\title{
Detection of bacterial species involved in perimplantitis concerned with cultural and RT-PCR
}

\author{
Marcello Gatti, Giovanna Costa, Tatiana Giulia Rizzati, Francesca Scandurra \\ Dipartimento di Scienze Odontostomatologiche, sezione di Microbiologia, Università degli Studi di Bologna.
}

Key words: periodontitis, implant dentistry, cultural, RT-PCR

Rilevazione di specie batteriche implicate nelle perimplantiti con esame colturale e RT-PCR

\section{SUMMARY}

Dental implants offer new treatment options for edentulous either partially or completely, now represent a viable alternative to conventional fixed protheses.

Dental implants are colonized by a flora dominated by Gram-positive facultative aerobic, while in patients with bone loss and formation of pockets peri-implant diseases was found a significant difference in the composition of microflora, bacteria, Gram-negative anaerobes in particular Fusobacterium spp., Treponema denticola (Spirochetes), Tannerella forsythensis, Aggregatibacter actinomycetemcomitans, Prevotella intermedia as interim black-pigmented bacteria, Porphyromonas gingivalis, often in high concentrations.

Aims. The purpose of this study was to identify those at risk of perimplantitis using 2 techniques: RT-PCR examination of trade and culture. The results were compared taking into consideration the advantages and disadvantages of both methods.

Materials and methods. We studied 24 patients ( 14 women and 10 men), aged, women between 43 and 76 years, with an average of $63.8+/-10.9$ years, men between 45 and 88 years with a average of 64.3 years $+/-12.5$ years.

Was performed a double levy of sub-gingival plaque at multiple sites that had an implant CAL (clinical attachment level) $>4 \mathrm{~mm}$ in order to assess the microbiological identification with the two techniques: Examining culture and Real-Time PCR of Commerce (Gum-Sunstar) that identifies 4 bacterial species: A. actinomycetemcomitans (A.a.), P.gingivalis (P.g.), T.forsythensis (T.f.), and T.denticola (T.d.).

Results. All patients studied were positive to both tests with charger high: the consideration of tenure, with CFU / ml > $10^{5}$, was positive in $66.6 \%$ of samples by:T.f., and P.g., in $12.5 \%$ for A.a., while T.d. not been sought by examining culture, the RT-PCR was positive, with high loads, in $95.8 \%$ of samples for T.f., in $79.1 \%$ for P.g., in $12.5 \%$ for A.a. and $20.8 \%$ for T.d. The test crop showed the presence of even P.intermedia in $91.6 \%$ of the samples, as well as Capnocytophaga spp. in $54.1 \%$, F.nucleatum spp. in $50 \%$ and Campylobacter spp. $25 \%$.

Conclusions. The data show that RT-PCR has greater sensitivity than culture examination as well as response times are in favor of RT-PCR, but the kits Trade identify a limited number of species present no bacterial resistance if it were taking antibiotics. There are several factors (genetic, environmental and systemic diseases) of the subject that may affect the results of microbiological contamination. It is perhaps for this reason that many dentists often consider the microbiological examination of "unsafe" because there are not always matching the survey clinical microbiological examination then the same situation microbiological not always the same clinical situation. Being able to pinpoint those responsible for periodontal infection and inflammation, however, offers the opportunity to vary quite rightly, on the basis of virulence factors and evaluation of bacteria, the timing of recalls for the TPS (periodontal therapy support), leaving as a last choice antibiotic therapy targeted.

\section{INTRODUZIONE}

L'implantologia orale osteointegrata ha rinnovato radicalmente la pratica odontoiatrica nel campo delle riabilitazioni protesiche. Gli impianti dentali offrono nuove possibilità di trattamento per le edentulie sia parziali che totali, rappresentando oggi una valida alternativa alla protesi fissa tradizionale. Le cause di fallimento degli impianti $(8,9)$ possono essere diverse tra cui le infezioni sostenute da batteri Gram negativi anaerobi, come nella gengivite e parodontite.

Gli impianti osteointegrati con successo sono colonizzati da una flora predominata da batteri Gram positivi aerobi facoltativi, la quale non mostra cambiamenti significativi nella composizione sopra i cinque anni negli impianti clinicamente stabili, mentre nei pazienti con perdita di osso e formazione di tasche periimplantari è stata trovata una notevole differenza nella composizione della microflora: batteri Gram negativi anaerobi $(7,11,13,14,17)$, in particolare Fusobacterium spp., Spirochete, Tannerella forsythensis, Aggregatibacter actinomycetemcomitans, microorganismi nigropigmenti come Prevotella intermedia, Porphyromonas gingivalis e spesso in alte concentrazioni (11).

La periimplantite è un processo infiammatorio della mucosa e dell'osso periimplantare che determina una perdita discreta del tessuto di supporto nei siti implantari colpiti $(1,15)$. Questa è la ragione per cui di fronte ad un caso di periimplantite non possiamo parlare di fallimento implantare, il cui segno cardine è la presenza di mobilità implantare (3), esito anatomopatologico di un processo che ha condotto alla completa perdita dell'interfaccia osso-impianto (10).
La rimozione regolare e attenta della placca sopragengivale non ha solo l'effetto di migliorare i parametri associati all'infiammazione delle gengive, ma anche quello di modulare la composizione della placca sottogengivale.

Le tradizionali colture di batteri anaerobi, che sono state fino ad oggi il metodo più utilizzato per l'identificazione dei batteri parodontopatogeni, hanno riscontrato diversi problemi sia di trasporto per i batteri che spesso arrivavano in laboratorio già morti, sia da un punto di vista metodologico di laboratorio dove diventa molto difficile la crescita in terreni di coltura e la successiva identificazione; proprio per questo motivo l'esame colturale è stato ormai sostituito dalla RealRime PCR che è sicuramente una metodica più facile e veloce ma soprattutto più sensibile (4).

\section{MATERIALI E METODI}

Sono stati studiati 24 pazienti (14 donne e 10 uomini), di età compresa , le donne fra 43 e 76 anni, con una media di 63.8 +/- 10.9 anni, gli uomini tra 45 e 88 anni, con una media di 64.3 anni $+/-12.5$ anni.

Tutti i pazienti hanno perduto elementi dentari per parodontopatia: essi sono stati in parte sostituiti con implantoprotesi (impianti presenti 44 nei cavi orali delle donne e 36 in quelle degli uomini). La media temporale degli impianti e della loro attività era di 11.5 anni nelle donne e di 10.7 anni negli uomini. Con l'utilizzo della sonda parodontale PCP 15 della Hu- Friedy sono stati valutati parametri clinici come BOP (sanguinamento al sondaggio), la presenza di essudato, la profondità delle tasche parodontali (PPD), il livello di attac-

\section{Corresponding author: Marcello Gatti}

Università degli Studi di Bologna, Dipartimento di Scienze Odontostomatologiche, sezione di Microbiologia

40 I 25 Bologna, Italia - Via San Vitale, 59 - Tel: +39 05। 2088I56 - Fax 05। 225208

E mail: marcello.gatti@unibo.it 
co clinico (CAL), la mobilità (MOB). Sono stati valutati dati anamnestici come la familiarità per la malattia parodontale, la condizione di fumatori, la presenza di malattie metaboliche, di ipertensione o di altri problemi sistemici. È stata considerata la frequenza abituale della terapia di supporto (per tutti > di un anno) e l' utilizzo domiciliare dei presidi per l'igiene degli spazi interprossimali come riportato nella Tabella 1.

Tabella I. Caratteristiche dei pazienti studiati.

\begin{tabular}{|c|c|c|}
\hline & Donne & Uomini \\
\hline $\mathbf{N}^{\circ}$ pazienti & 14 & 10 \\
\hline Età Media & $63,8+/-10,9$ anni & $64,3+/-12,5$ anni \\
\hline Range & Da 43 a 76 & $\mathrm{Da} 45$ a 88 \\
\hline $\mathrm{N}^{\circ}$ totale impianti presenti & 44 & 36 \\
\hline \multicolumn{3}{|l|}{ Media temporale della presenza } \\
\hline degli impianti nel cavo orale & II,5 anni & 10,7 anni \\
\hline $\mathrm{N}^{\circ} \mathrm{pz}$ con $\mathrm{BOP}>20 \%$ & 11 & 4 \\
\hline $\mathrm{N}^{\circ}$ siti CAL $>4 \mathrm{~mm}$ & $>4$ siti & $>4$ siti \\
\hline Familiarità & $1 \mathrm{I} / 14$ & $4 / 10$ \\
\hline \multicolumn{3}{|l|}{ Frequenza abituale terapia } \\
\hline di supporto & $>$ I anno & $>I$ anno \\
\hline \multicolumn{3}{|l|}{ Utilizzo domiciliare presidi per } \\
\hline l'igiene degli spazi interprossimali & $5 / 14$ & $2 / 10$ \\
\hline $\mathrm{N}^{\circ} \mathrm{pz}$ fumatori & $4,<10$ sigarette/die & I (pipa) \\
\hline $\mathrm{N}^{\circ} \mathrm{pz}$ ipertesi & 4 di cui I in TAO & 3 \\
\hline $\mathrm{N}^{\circ} \mathrm{pz}$ diabetici (tipo 2) & 3 & 0 \\
\hline $\mathrm{N}^{\circ} \mathrm{pz}$ con altre patologie sistemiche & 0 & 0 \\
\hline
\end{tabular}

Nella Tabella 2 sono riportati i criteri per la non inclusione nello studio.

\section{Tabella 2.}

I. Avere assunto antibiotici nei tre mesi precedenti il prelievo.

2. Avere utilizzato collutori antibatterici nell'ultima settimana prima del prelievo.

3. Essersi sottoposti a terapia di supporto (causale) durante i tre mesi precedenti il prelievo.

4. Presenza di gravidanza.

Dopo aver inquadrato clinicamente il paziente per lo studio, è stato eseguito un doppio prelievo di placca sub-gengivale in più siti implantari che avessero un CAL (livello di attacco clinico) $>4 \mathrm{~mm}$ al fine di valutare il profilo microbiologico con le due tecniche identificative: esame colturale e Real-Time PCR.

Nella Tabella 3 sono riportate le modalità utilizzate per il prelievo del materiale patologico.

Tabella 3.

I. Isolare il campo operatorio.

2. Asciugare il sito individuato per il prelievo.

3. Inserire 2 coni di carta sterili (uno per l'esame colturale e l'altro per la RT-PCR), nei siti con sondaggio > $4 \mathrm{~mm}$ per 20'.

4. Inserire i coni nelle provette sterili con terreno di trasporto per l'esame colturale e senza terreno di trasporto per RT-PCR.

5. Inviare i campioni nei rispettivi laboratori.

Sono stati selezionati 4 siti di prelievo per ogni paziente, mediante 2 coni di carta sterili, per sito, che venivano inseriti in provette per RT-PCR e con terreno di trasporto per l'esame colturale.

I campioni sono stati processati per la ricerca utilizzando i protocolli in vigore in laboratorio di Microbiologia.

Il primo protocollo prevede un esame microscopico a fresco per evidenziare batteri mobili, la presenza di aggregati batterici adesi a cellule della mucosa, cocchi, batteri filamentosi, blastospore di lievito, globuli bianchi, globuli rossi, ecc. e in campo oscuro per evidenziare la presenza di spirochete.

Nella Figura I si può vedere un preparato microscopico a fresco in campo oscuro, ingrandimento 400x, dove si può vede- re la presenza di 2 cellule della mucosa orale e la presenza di batteri adesi alle stesse e spirochete libere.

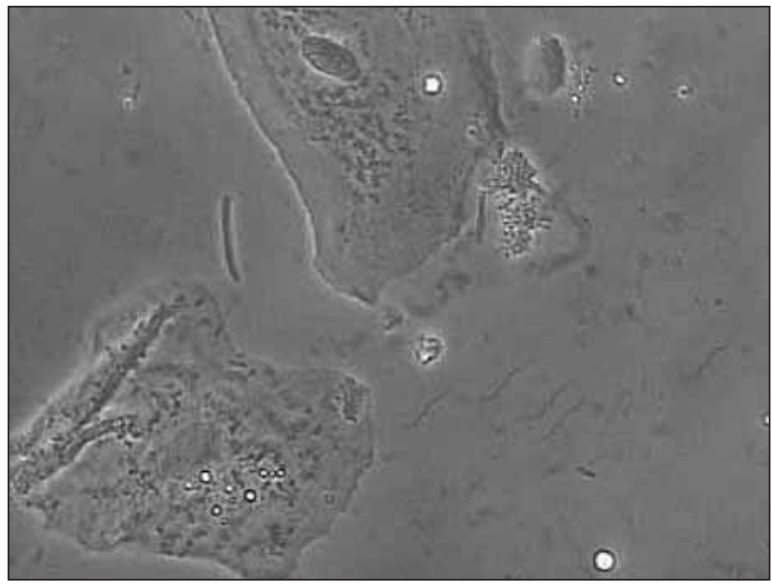

Figura I

Dal risultato di queste osservazioni si decideva la scelta di terreni diversi per l'esame colturale come piastre di: Herellea agar (Biolife), Mannitol salt agar (Bioloife), MTS agar (biolife), agar sangue di cavallo (Biolife), Brucella agar (Biolife), CNA agar (Biolife), agar blood Schaedler (Biolife), GC-Lect agar (BD) e/o altri terreni selettivi preparati in laboratorio, come prevedono i protocolli per l'esame colturale per batteri aerobi, microaerofili ed anaerobi in uso in laboratorio.

Per la semina è stata utilizzata un ansa calibrata monouso sterile da $10 \mu \mathrm{l}$. L'ansa veniva strisciata sulle piastre in 4 settori diversi, per ottenere una conta semiquantitativa finale di $\geq 10^{5} \mathrm{CFU} / \mathrm{ml}$. Le piastre venivano incubate a $37^{\circ} \mathrm{C}$ in aerobiosi per 48-72 ore, in microaerofilia e in anaerobiosi per 7-15 giorni utilizzando Gen bag (bioMérieux). Le colonie sono state isolate utilizzando gli stessi terreni di crescita ed identificate con i sistemi Api (bioMérieux).

Per l'antibiogramma (ABG) sono state utilizzate piastre di Brucella agar (Biolife) utilizzando il metodo di Kirby-Bauer ed incubati a $37^{\circ} \mathrm{C}$ in aerobiosi, microaerofilia ed anaerobiosi a seconda dei batteri isolati. Gli antibiotici scelti per il saggio sono stati: amoxicilllina + ac. clavulanico (AMC), metronidazolo (MTZ), tetracicline (TE), clindamicina (CC), rifampicina (RD), ciprofloxacina (CIP) e ceftriaxone (CRO).

Per l'analisi molecolare i prelievi da esaminare sono stati inviati, tramite la ditta GUM, la quale si avvale della collaborazione dell'istituto IAI (Instut fur Angewandte Immunologie), di Zuchwil, in Svizzera.

\section{RISULTATI.}

Tutti i pazienti studiati sono risultati positivi al test con cariche batteriche $\geq 10^{5} \mathrm{CFU} / \mathrm{ml}$. In particolare, l'esame colturale, con $\geq 10^{5} \mathrm{CFU} / \mathrm{ml}$, è risultato positivo per: Tannerella forsythensis (T.f.) in 16/24 campioni (66.6\%), 23/24 con RTPCR (95.8\%), Porphyromonas gingivalis (P.g.) in 16/24 campioni (66.6\%), contro 19 (79.1\%) con RT-PCR, Aggregatibacter actinomycetemcomitans (A.a.) in 3/24 campioni (12.5\%) contro 5/24 (20.8\%) con RT-PCR., Treponema denticola (T.d.) non è stata cercata con l'esame colturale, ma $16 / 24$ (66.6\%) preparati microscopici in campo oscuro sono risultati positivi per spirochete, mentre 18/24 campioni (75.0\%) sono risultati positivi con RT-PCR, come riportato nella Figura II.

L'esame colturale ha permesso di identificare la presenza anche di altri batteri, sempre con carica $\geq 10^{5} \mathrm{CFU} / \mathrm{ml}$, come: 22/24 campioni positivi per P.intermedia (P.i.) (91.6\%), 13/24 campini positivi per Capnocytophaga spp.(Capno.) (54.1\%), 12/24 campioni positivi per F.nucleatum (F.n.) (50\%) e 6/24 campioni positivi per Campylobacter spp. (Campy.) (25\%) come riportato nella Figura III. 


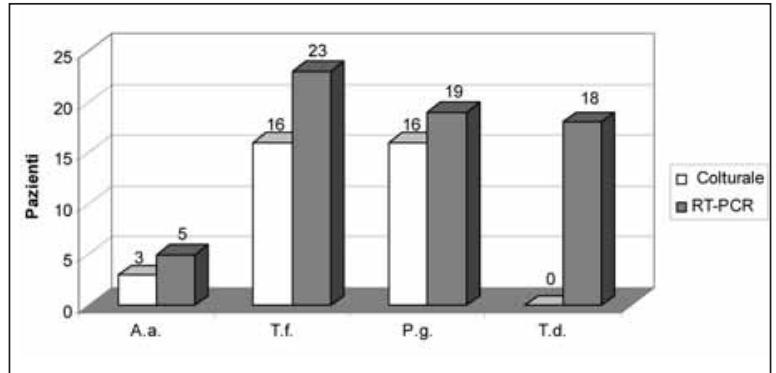

Figura II

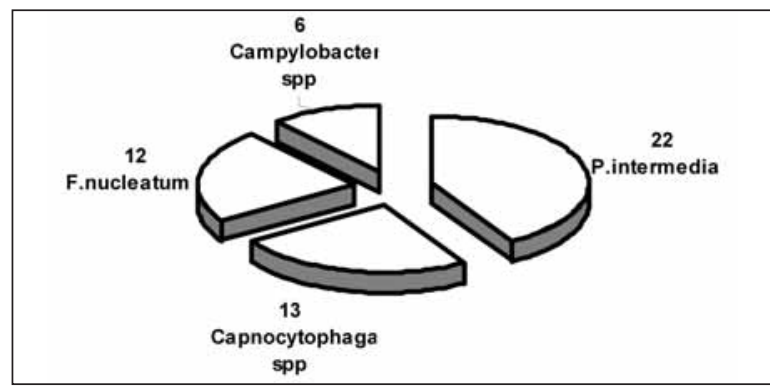

Figura III

L'ABG ha dimostrato resistenze varie, in particore l'amoxicillina + acido clavulanico e il metronidazolo verso P.intermedia, P.gingivalis e T.forsythensis, come riportato nella Tabella 4

\section{Tabella 4}

\begin{tabular}{lcccccccc}
\hline Batteri & $\mathbf{N}^{\circ}$ ceppi & AMC & MTZ & RD & TE & CC & CRO & CIP \\
\hline A. a. & $\mathbf{3}$ & 0 & 0 & 0 & 0 & 0 & I & I \\
\hline P. i. & $\mathbf{2 2}$ & 10 & 7 & 4 & 4 & 4 & 6 & 2 \\
\hline P. g. & 16 & 12 & II & 5 & 8 & 5 & 7 & 3 \\
\hline F. n. & 12 & 8 & 2 & 0 & I & I & I & I \\
\hline T. f. & 16 & 9 & 9 & 5 & 7 & 3 & 3 & 2 \\
\hline Capno. & 13 & 4 & 5 & 5 & 4 & 3 & 3 & 2 \\
\hline Campy. & $\mathbf{6}$ & 4 & 3 & 0 & 4 & 2 & 6 & I \\
\hline
\end{tabular}

\section{DISCUSSIONE}

I dati mostrano che RT-PCR ha una sensibilità maggiore rispetto all'esame colturale così come i tempi di risposta sono a favore di RT-PCR, ma i kit del commercio identificano un numero limitato di specie e non evidenziano resistenze batteriche nel caso si dovesse intraprendere terapia antibiotica.

Risulta importante monitorare e indirizzare la composizione del microbiota presente nei solchi e nelle tasche perimplantari per garantire successo e non sopravvivenza a questi manufatti.

La placca dentale può provocare condizioni patologiche se non attentamente controllata.

Il biofilm presente nel cavo orale è una struttura molto complessa, al cui interno, numero, proporzioni e prevalenza dei patogeni non è dipendente solo dalla sua quotidiana dissociazione meccanica (uso di spazzolino), ma legata anche all'habitat nel quale si trova e alla risposta dell'ospite.

Ci sono dunque fattori genetici (2) ambientali (fumo ad es.), malattie sistemiche del soggetto che condizionano il trattamento o comunque i risultati dello scardinamento dei legami batterici. È forse per questo motivo che molti odontoiatri spesso considerano l'esame microbiologico "poco sicuro", perché non sempre ci sono corrispondenze: a una medesima situazione microbiologica non sempre corrisponde la medesima situazione clinica perché bisogna fare i conti con ospiti differenti, che hanno fattori genetici, situazioni di salute generale e fattori ambientali differenti.

Credo però che, attraverso l'esame microbiologico effettuato per valutare la risposta della comunità batterica nella varia- zione delle sue proporzioni, e possibili mancate corrispondenze da esso evidenziate a seguito di trattamento, si possano portare alla luce problematiche dell'habitat e dell'ospite. Sarà possibile, quindi, organizzare un trattamento mirato, per quanto riguarda tempi e modi, che tenga conto di queste individualità, forse prevenendo la conclamata situazione clinica di infiammazione o infezione causata dalla presenza patogena. I dati provenienti da questa ulteriore indagine stimolano inoltre la motivazione del paziente ad effettuare una scrupolosa igiene orale domiciliare anche sulla base delle più recenti acquisizioni in merito alle potenzialità infettive dei batteri parodontopatogeni in altri distretti corporei tramite il circolo sanguigno (5).

Il fatto di poter individuare esattamente i responsabili dell'infiammazione e dell'infezione parodontale offre l'opportunità di variare a ragion veduta, sulla base dei fattori di virulenza e della valutazione della carica batterica, i tempi dei richiami per la TPS (terapia parodontale di supporto), lasciando come ultima scelta la terapia antibiotica $(6,16)$.

Bisogna comunque tener presente che siamo di fronte a strutture altamente complesse da un punto di vista microbiologico e questa complessità permette di affermare con sicurezza che dopo il trattamento la tendenza sarà verso il ritorno ad una aggregazione batterica relativamente simile, anche se con una auspicabile eliminazione o riduzione delle specie patogene.

\section{BIBLIOGRAFIA}

1. Albrektsson T, Isidor F. Consensus report of session IV. In: Lang NP, Karring $\mathrm{T}$ ed. Proceedings of the First European Workshop on Periodontology. Quintessence 1994; 365-9.

2. Ebersole JL, Taubman MA, Smith DJ. Local antibody responses in periodontal disease. J Periodontol 1985; 56(11 suppl): 51-5.

3. Esposito M, Thomsen P, Ericson LE, Lekholm U. Histopathologic observations on early oral implant failures. Int J Oral Maxillofac Implants. 1999 Nov-Dec;14(6): 798-810.

4. Gatti M. Isolamento di batteri nella malattia parodontale. Poster presentato al XXXVII Congresso Nazionale AMCLI. Stresa 6-8 ottobre 2008. Abstract 035.

5. Genco R, Offenbacher S, Bech J. Periodontal disease and cardiovascular disease: epidemiology and possible mechanisms. J A Dent Assoc 2002; 133 (suppl): 14-22.

6. Haffajee AD, Socransky SS, Lindhe J. Effetti della terapia sui microgranismi della regione dento-gengivale in: Parodontologia Clinica e odontoiatria implantare (cap.26), Edi Ermes 2006.

7. Henderson B, Wilson M, Sharp L, Ward JM. Actinobacillus actinomycetemcomitans. J Med Microbiol 2002; Dec 51(12): 1013-20.

8. Lang NP, Wilson TG, Corbet EF. Biological complications with dental implants: their prevention, diagnosis and treatment. Clin Oral Impl Res 2000; 11 (Suppl.), 146-55.

9. Leonhardt A, Renvert S, Dahlén G. Microbial findings at failing implants. Clin Oral Impl Res 1999; 10, 339-45.

10. Mombelli A, Lang NP. The diagnosis and treatment of periimplantitis. Periodontol 2000. 1998; 17, 68-76.

11. Mombelli A, Van Oosten MAC, Schürch E, Lang NP. The microbiota associated with successful or failing osseointegrated titanium implants. Oral Microbiol Immunol 1987; 2: 145-51.

12. Mombelli A. Microbiology of the dental implant. Adv Dent Res. 1993Aug; $7(2): 202-6$

13. Rodemburg JP, van Winkelhoff AJ, Winkel EG, et al. Occurrence of Bacteroides gingivalis, Bacteroides intermedius and A. actinomycetemcomitans in severe periodontitis in relation to age and treatment history. $J$ Clin Periodontol 1990; 17(6): 392-99.

14. Slots J, Ting M, Adams DA, Caton J, et al. A. actinomycetemcomitans and Porphyromonas gingivalis in human periodontal disease: occurrence and treatment. Periodontol 2000. 1999; 20: 82-112.

15. Smith DE, Zarb G. Criteria for success of osseointegrated endosseous implants. J Prosth Dent 1989; 62, 567-72.

16. Socransky SS, Haffajee AD. Microbial mechanism in the pathogenesis of destructive periodontal diseases: a critical assesment. J Periodontol Res 1991; 26: 195-21.

17. Van Winkelhoff AJ, Wolf JW. Actinobacillus actinomycetemcomitansassociated peri-implantitis in an edentulous patient. A case report. J Clin Periodontol 2000 Jul; 27(7): 531-5. 